Bulletin of the Institute of Mathematics

Academia Sinica (New Series)

Vol. 12 (2017), No. 2, pp. 125-139

DOI: 10.21915 /BIMAS.2017202

\title{
NOTES ON LOCALLY FREE CLASS GROUPS
}

\author{
CHIA-FU YU
}

\begin{abstract}
Institute of Mathematics, Academia Sinica and NCTS, 6th Floor, Astronomy Mathematics Building, No. 1, Roosevelt Rd. Sec. 4, Taipei, Taiwan, 10617.

E-mail: chiafu@math.sinica.edu.tw
\end{abstract}

\|\|$\|$

\begin{abstract}
A theorem of Swan describes the locally free class group of a maximal order in a central simple algebra over a number field. Swan's theorem was generalized by Curtis and Reiner to separable algebras for both number fields and global function fields. The proof given by Curtis and Reiner uses Algebraic $K$-Theory. Our motivation is to provide a proof which works for both number fields and global function fields and which does not rely on Algebraic K-Theory.
\end{abstract}

\section{Introduction}

A more precise title of this article is "Notes on locally free class groups of orders in separable algebras over global fields". A theorem of Swan states that the locally free class group of a maximal order in a central simple algebra over a number field is isomorphic to a restricted ideal class group of the center; see [11, Theorem 1, p. 56] or Theorem 4, Fröhlich [5] generalized Swan's theorem for any separable algebra over a number field using the adeles. For separable algebras over global function fields, this result was limited to those where the Eichler condition is satisfied; see [9, (35.14) Theorem p. 313]. The Eichler condition in the function field case was removed later by Curtis and Reiner; see [2, Theorem (49.32), p. 233]. This generalization is more applicable to integral representations of finite groups. The proof given by Curtis and Reiner uses Algebraic $K$-Theory while the original proof of Swan, which is much shorter, does not. As the statements of Swan's theorem and its generalization apparently are not involved with Algebraic $K$-Theory,

Received February 9, 2017.

AMS Subject Classification: 16H20, 11R52.

Key words and phrases: Ideal class group, lattice, order. 
it is natural to have an Algebraic- $K$-Theory-free proof as Swan's proof. This was the motivation of these notes.

These notes are organized as follows. In Section 2, we introduce the locally free class group of an $R$-order $\Lambda$ in a separable $K$-algebra, for a Dedekind domain $R$ with fraction field $K$. The locally free class groups can be defined in a more general setting. However, since results discussed here will be restricted to the case of global fields, we do not attempt to discuss its definition as general as possible. Instead, we illustrate the essential idea of this notion.

In Section 3 we discuss the main theorem (Theorem 34) on locally free class groups due to Curtis and Reiner. We then explain how Swan's theorem (Theorem 4) is deduced from the main theorem. The proof of Theorem 3 is given in Section 5, for which we follow closely the original proofs of Swan and Fröhlich. The strong approximation theorem (SAT) plays the key role in the proof and we give a short exposition in Section 4.

\section{The Cancellation Law}

Let us first motivate the notion of locally free class groups by the classical theorem of Steinitz. Let $R$ be a Dedekind domain with fraction field $K$ and assume that $R \neq K$. An $R$-lattice is a finite torsion-free $R$-module $M$, that is, $M$ is isomorphic to a finite $R$-submodule in a (finite-dimensional) $K$-vector space. We have the following well-known results concerning the classification of $R$-lattices (cf. [1, Theorem (4.13), p. 85]):

(1) Every $R$-lattice $M$ is $R$-projective, and $M \simeq \oplus_{i=i}^{n} J_{i}$ for some non-zero ideals $J_{i}$ of $R$, where $n$ is the $R$-rank of $M$.

(2) Two $R$-lattices $M=\oplus_{i=i}^{n} J_{i}$ and $M^{\prime}=\oplus_{i=i}^{m} J_{i}^{\prime}$ of the form in (1) are isomorphic if and only if $n=m$ and the products $J_{1} \cdots J_{n}$ and $J_{1}^{\prime} \cdots J_{n}^{\prime}$ are isomorphic.

From the statement (2) one can easily deduce the following result: If $M$ and $M^{\prime}$ are two $R$-lattices, then we have

$$
M \oplus R \simeq M^{\prime} \oplus R \Longleftrightarrow M \simeq M^{\prime}
$$

The property (2.1) is called the cancellation law. 
As is well known, a useful and easier way of studying rings is to study their modules, instead of studying their underlying ring structures. Based on this idea, the cancellation law then allows us to distinguish certain rings which share the same good properties. For example, consider the quaternion $\mathbb{Q}$-algebras $B_{p, \infty}$, which are those ramified exactly the two places $\{p, \infty\}$ of $\mathbb{Q}$, for various primes $p$. Choose a maximal order $\Lambda(p)$ in each $B_{p, \infty}$, that is, $\Lambda(p)$ is not strictly contained in another $\mathbb{Z}$-order in $B_{p, \infty}$. Then one can show that the cancellation law for ideals of $\Lambda(p)$ holds true if and only if $p \in\{2,3,5,7,13\}$. We will also give a proof of this fact (Proposition 5).

Now let $\Lambda$ be an (not necessarily commutative) $R$-algebra which is finitely generated as an $R$-module. The above example shows that the cancellation law for (right) projective $\Lambda$-modules need not hold in general. In Mathematics, we often encounter a situation that a desired nice property turns out to be impossible. In that situation one usually introduces a more flexible notion so that the desired nice property remains valid in the slightly weaker setting. For the present case, one can for example consider the following weaker equivalence relation:

Define $M \sim M^{\prime}$ if $M \oplus \Lambda^{r} \simeq M^{\prime} \oplus \Lambda^{r}$ for some integer $r \geq 0$.

Then it follows obviously from the definition that the cancellation law holds true for this new equivalence relation, that is, we have

$$
M \oplus \Lambda \sim M^{\prime} \oplus \Lambda \Longleftrightarrow M \sim M^{\prime}
$$

Two modules $M$ and $M^{\prime}$ satisfying the property (2.2) are said to be stably isomorphic.

The reader who is familiar with algebraic topology would immediately recognize that the way of defining a "stable" notion here is similar to that in the definition of stable homotopy groups. This is also similar to that in the definitions of stable freeness and stable rationality. These are reminiscent of the definition of groups $K_{0}$ and $K_{1}$ in Algebraic $K$-theory using an inductive limit procedure. 


\section{Locally Free Class Groups}

For the remainder of this article we assume that the ground field $K$ is a global field; that is, $K$ is a finite extension of $\mathbb{Q}$ or $\mathbb{F}_{p}(t)$. Thus, $R$ is the ring of $S$-integers of $K$ for a non-empty finite set of places $S$ containing all archimedean ones, if any. Let $A$ denote a finite-dimensional separable $K$-algebra. In other words, $A$ is a finite-dimensional semi-simple $K$-algebra such that the center $C$ of $A$ is a product of finite separable field extensions $K_{i}$ of $K$. Recall that an $R$-order in $A$ is an $R$-subring of $A$ which is finitely generated as an $R$-module and generates $A$ over $K$. We let $\Lambda$ denote an $R$ order in $A$. A $\Lambda$-lattice $M$ is an $R$-torsion free finitely generated $\Lambda$-module.

Example. Let $G$ be a finite group with char $K \nmid|G|$. Then the group algebra $A=K G$ is a separable $K$-algebra. We can see this by Maschke's Theorem (cf. [1, Theorem 3.14, p. 42]): Every finite-dimensional representation of $G$ over $K$ is a direct sum of irreducible representations. Then by definition $K G$ is a semi-simple $K$-algebra. Applying Maschke's Theorem again to an algebraic closure $\bar{K}$ of $K$, we see that the algebra $\bar{K} \otimes_{K} K G=\bar{K} G$ is also semi-simple. Therefore, $A$ is a separable $K$-algebra. Clearly, the group ring $\Lambda=R G$ is an $R$-order in $A$, and any representation $M$ of $G$ over $R$ is a $\Lambda$-lattice.

For any integer $n \geq 1$, denote by $\operatorname{LF}_{n}(\Lambda)$ the set of isomorphism classes of locally free right $\Lambda$-modules of rank $n$. Two locally free right $\Lambda$-modules $M$ and $M^{\prime}$ are said to be stably isomorphic, denoted by $M \sim_{s} M^{\prime}$, if

$$
M \oplus \Lambda^{r} \simeq M^{\prime} \oplus \Lambda^{r}
$$

as $\Lambda$-modules for some integer $r \geq 0$. The stable class of $M$ will be denoted by $[M]_{s}$, while the isomorphism class is denoted by $[M]$. By a $\Lambda$-ideal we mean a $\Lambda$-lattice in $A$, that is, it is an $R$-lattice which is also a $\Lambda$-module. Let $\mathrm{Cl}(\Lambda)$ denote the set of stable classes of locally free right $\Lambda$-ideals in $A$. The Jordan-Zassenhaus Theorem (cf. [1, Theorem 24.1, p. 534]) states that $\operatorname{LF}_{1}(\Lambda)$ is a finite set, and hence so the set $\mathrm{Cl}(\Lambda)$ is. We define the group structure on $\mathrm{Cl}(\Lambda)$ as follows. Let $J$ and $J^{\prime}$ be two locally free $\Lambda$-ideals. Define

$$
[J]_{s}+\left[J^{\prime}\right]_{s}=\left[J^{\prime \prime}\right]_{s}
$$


where $J^{\prime \prime}$ is any locally free $\Lambda$-ideal satisfying

$$
J \oplus J^{\prime}=J^{\prime \prime} \oplus \Lambda
$$

as $\Lambda$-modules. Such a $\Lambda$-ideal $J^{\prime \prime}$ always exists and we will see this in Section 5 .

Lemma 1. The finite set $\mathrm{Cl}(\Lambda)$ with the binary operation defined in (3.1) forms an abelian group.

Proof. By (3.2), the commutativity holds true. We prove the associativity. Let $J_{1}, J_{2}, J_{3}$ be three locally free ideals of $\Lambda$. Suppose we have $\left[J_{1}\right]_{s}+\left[J_{2}\right]_{s}=$ $\left[J^{\prime}\right]_{s}$ and $\left[J^{\prime}\right]_{s}+\left[J_{3}\right]_{s}=\left[J^{\prime \prime}\right]_{s}$. Then

$$
\left(J_{1} \oplus J_{2}\right) \oplus J_{3} \simeq \Lambda \oplus J^{\prime} \oplus J_{3} \simeq J^{\prime \prime} \oplus \Lambda^{2} .
$$

Similarly if $\left[J_{2}\right]_{s}+\left[J_{3}\right]_{s}=\left[G^{\prime}\right]_{s}$ and $\left[J_{1}\right]_{s}+\left[G^{\prime}\right]_{s}=\left[G^{\prime \prime}\right]_{s}$, then $J_{1} \oplus\left(J_{2} \oplus J_{3}\right) \simeq$ $G^{\prime \prime} \oplus \Lambda^{2}$. This shows $\left[J^{\prime \prime}\right]_{s}=\left[G^{\prime \prime}\right]_{s}$ and the associativity holds true.

Definition 2. The group $\mathrm{Cl}(\Lambda)$ with group law defined as in (3.1) is called the locally free class group of $\Lambda$.

We introduce some more notation. Denote by $C$ the center of $A$. One has $C=\prod_{i}^{s} K_{i}$ and $A=\prod_{i}^{s} A_{i}$, where each $A_{i}$ is a central simple algebra over $K_{i}$. For any place $v$ of $K$, let $K_{v}$ denote the completion of $K$ at $v$, and $O_{v}$ the valuation ring if $v$ is non-archimedean. We also write $R_{v}$ for $O_{v}$ when $v \notin S$. Let $A_{v}:=K_{v} \otimes_{K} A, C_{v}:=K_{v} \otimes_{K} A$ and $\Lambda_{v}:=R_{v} \otimes_{R} \Lambda$ be the completions of $A, C$ and $\Lambda$ at $v$, respectively. By a place $w$ of $C$ we mean a place $w$ of $K_{i}$ for some $i$; we say that the algebra $A$ splits (resp. is ramified) at the place $w$ of $C$ if $A_{i}$ splits (resp. is ramified) at the place $w$. Let $\widehat{R}=\prod_{v \notin S} R_{v}$ be the profinite completion of $R$, and let $\widehat{K}=K \otimes_{R} \widehat{R}$ be the finite $S$-adele ring of $K$; one also writes $\mathbb{A}_{K}^{S}$ for $\widehat{K}$. Put $\widehat{A}:=\widehat{K} \otimes_{K} A$, $\widehat{C}:=\widehat{K} \otimes_{K} C$ and $\widehat{\Lambda}:=\widehat{R} \otimes_{R} \Lambda=\prod_{v \notin S} \Lambda_{v}$.

It is a basic fact that the set $\operatorname{LF}_{1}(\Lambda)$ is isomorphic to the double coset space $A^{\times} \backslash \widehat{A}^{\times} / \widehat{\Lambda}^{\times}$. There is a natural surjective map

$$
\operatorname{LF}_{1}(\Lambda) \rightarrow \mathrm{Cl}(\Lambda)
$$

by sending $[J] \mapsto[J]_{s}$. Let $N_{A_{i} / K_{i}}: A_{i} \rightarrow K_{i}$ denote the reduced norm map. It induces a surjective map $N_{i}: \widehat{A}_{i}^{\times} \rightarrow \widehat{K}_{i}^{\times}$because the norm map 
$A_{i} \otimes K_{v} \rightarrow K_{i} \otimes K_{v}$ is surjective for any finite place $v$ of $K$. The reduced norm map $N: A=\prod_{i} A_{i} \rightarrow C=\prod_{i} K_{i}$ is simply defined as the product $N=\left(N_{A_{i} / K_{i}}\right)_{i}$. Then we have a surjective map $N: \widehat{A}^{\times} \rightarrow \widehat{C}^{\times}$, and it gives rise to the surjective map (again denoted by)

$$
N: \mathrm{LF}_{1}(\Lambda) \simeq A^{\times} \backslash \widehat{A}^{\times} / \widehat{\Lambda}^{\times} \rightarrow N\left(A^{\times}\right) \backslash \widehat{C}^{\times} / N\left(\widehat{\Lambda}^{\times}\right) .
$$

We will see that $N\left(A^{\times}\right)=C_{+, A}^{\times}$, where

$$
C_{+, A}^{\times}:=\left\{a \in C^{\times} \mid r(a)>0 \text { for all real places } r \text { ramified in } A\right\} .
$$

The main theorem for the locally free class groups is as follows [2, Theorem (49.32), p. 233].

Theorem 3 (Curtis-Reiner). The map 3.4 factors through $\mathrm{LF}_{1}(\Lambda) \rightarrow$ $\mathrm{Cl}(\Lambda)$ and it induces an isomorphism of finite abelian groups

$$
\nu: \operatorname{Cl}(\Lambda) \simeq \widehat{K}^{\times} / C_{+, A}^{\times} N\left(\widehat{\Lambda}^{\times}\right) .
$$

We now describe Swan's theorem on locally free class groups. Assume that $A$ is a central simple algebra and $\Lambda$ is a maximal $R$-order in $A$. Define the ray class group $\mathrm{Cl}_{A}(R)$ of $K$ by

$$
\mathrm{Cl}_{A}(R):=I(R) / P_{A}(R)
$$

where $I(R)$ be the ideal group of $R$ and $P_{A}(R)$ be the subgroup generated by the principal ideals ( $a$ ) for $a \in K_{+, A}^{\times}$. Here $K_{+, A}^{\times} \subset K^{\times}$is the subgroup of $K^{\times}$defined as (3.5). In terms of the adelic language, the group $\mathrm{Cl}_{A}(R)$ is nothing but the group $\widehat{K}^{\times} / K_{+, A}^{\times} \widehat{R}^{\times}$.

Theorem 4 (Swan [11]). Let $K$ be a global field and $R$ the ring of $S$-integers of $K$ for a non-empty finite set of places $S$ containing all archimedean ones. Let $A$ be a central simple algebra and $\Lambda$ a maximal $R$-order in $A$. Then theres is an isomorphism of finite abelian groups $\mathrm{Cl}(\Lambda) \simeq \mathrm{Cl}_{A}(R)$.

To see Theorem 4 is an immediate consequence of Theorem 3 , we just need to check that $N\left(\Lambda_{v}^{\times}\right)=R_{v}^{\times}$for $v \notin S$ ( $\Lambda_{v}$ here is a maximal $R_{v}$-order). It is known that there exists a maximal subfield $E \subset A_{v}$ which is unramified over $K_{v}$. Since any two maximal orders in $A_{v}$ are conjugate, $\Lambda_{v}$ contains 
a copy of the ring of integers $O_{E}$ of $E$. As $E$ is unramified over $K_{v}$, the successive approximation shows that $N_{E / K_{v}}\left(O_{E}^{\times}\right)=R_{v}^{\times}$. It follows that $N\left(\Lambda_{v}^{\times}\right)=R_{v}^{\times}$.

Proposition 5. Let $B_{p, \infty}$ be the quaternion $\mathbb{Q}$-algebra ramified exactly at $\{p, \infty\}$, and $\Lambda(p)$ a maximal order in $B_{p, \infty}$ with a prime $p$. be as in Section 2. Then the cancellation law for ideals of $\Lambda(p)$ holds true if and only if $p \in$ $\{2,3,5,7,13\}$.

Proof. The cancellation law holds if and only if the map $\operatorname{LF}_{1}(\Lambda(p)) \rightarrow$ $\mathrm{Cl}(\Lambda(p))$ is bijective. By Swan's theorem, the locally free class group $\mathrm{Cl}(\Lambda(p)) \simeq$ $\mathrm{Cl}_{B_{p, \infty}}(\mathbb{Z})$ is trivial. Thus, the cancellation law holds true if and only if the class number $h(\Lambda(p))=\left|\mathrm{LF}_{1}(\Lambda(p))\right|$ is one. On the other hand we have the class number formula [3]

$$
h(\Lambda(p))=\frac{p-1}{12}+\frac{1}{3}\left(1-\left(\frac{-3}{p}\right)\right)+\frac{1}{4}\left(1-\left(\frac{-4}{p}\right)\right),
$$

where $(\dot{\bar{p}})$ denotes the Legendre symbol. From this one easily sees that $h(\Lambda(p))=1$ if and only if $p \in\{2,3,5,7,13\}$.

Remark 6. The cancellation law has been investigated by Vignéras [15] for general definite quaternion algebras over totally fields $F$. She showed that the cancellation law holds only when $[F: \mathbb{Q}]<33$ and that there are only finitely many orders can occur. The bound of degree was reduced significantly to 6 by Hallouin and Marie [6] and they gave a complete list of all definite Eichler orders that satisfy the cancellation property. The list is re-examed by Smertnig [10] due to an overlook on Vignéras' criterion.

For the remainder of this section we give a proof of the following basic fact.

Lemma 7. Let $A$ is a separable $K$-algebra and $C$ its center. Then $N\left(A^{\times}\right)=$ $C_{+, A}^{\times}$.

Proof. Since $A=\prod_{i} A_{i}$ and $C_{+, A}^{\times}=\prod_{i} K_{i,+, A_{i}}^{\times}$, it suffices to show $N\left(A^{\times}\right)=$ $K_{+, A}^{\times}$for any central simple $K$-algebra $A$. We can use the Hasse-Schilling norm theorem (the local-global principle for the reduced norm map) to describe $N\left(A^{\times}\right)$:

$$
N\left(A^{\times}\right)=\left\{x \in K^{\times} ; x \in N\left(A_{v}^{\times}\right) \forall v\right\} ;
$$


see [9, (32.9) Theorem, p. 275] and [9, (32.20) Theorem, p. 280]. Clearly $N\left(A_{v}^{\times}\right)=K_{v}^{\times}$when $v$ is complex, non-archimedean, or a real split place for $A$. It remains to show that if $v$ is a real ramified place for $A$, then one has $v(a)>0$ if and only if $a \in N\left(A_{v}^{\times}\right)$. This is given in the next lemma.

Lemma 8. Let $\mathbb{H}$ be the real Hamilton quaternion and $n \in \mathbb{N}$. Then $N\left(\mathrm{GL}_{n}(\mathbb{H})\right)=\mathbb{R}_{+}$.

Proof. We give two proofs of this result. One is topological and the other one is algebraic. The algebraic proof will be given in Lemma 9 .

Since $\left.\mathrm{GL}_{n}(\mathbb{H})\right)$ contains the subgroup $\left\{\operatorname{diag}(1, \ldots, a) \mid a \in \mathbb{H}^{\times}\right\}$, one has $N\left(\mathrm{GL}_{n}(\mathbb{H})\right) \supset N\left(\mathbb{H}^{\times}\right)=\mathbb{R}_{+}$. Thus, it suffices to show the other inclusion $N\left(\mathrm{GL}_{n}(\mathbb{H})\right) \subset \mathbb{R}_{+}$.

Note that the set $\mathrm{GL}_{n}(\mathbb{H})^{s s} \subset \mathrm{GL}_{n}(\mathbb{H})$ of semi-simple elements is open and dense in the classical topology. By continuity it suffices to show $N(x)>$ 0 for any $x \in \mathrm{GL}_{n}(\mathbb{H})^{s s}$. Since any such $x$ is contained in a maximal commutative semi-simple subalgebra, which is isomorphic to $\mathbb{C}^{n}$, we have $N(x)>0$ for $x \in \in \mathrm{GL}_{n}(\mathbb{H})^{s s}$.

Lemma 9. Let $D$ be a central division algebra over any field $K$ and $n \in \mathbb{N}$. Then $N\left(\operatorname{GL}_{n}(D)\right)=N\left(D^{\times}\right)$.

Proof. We give two proofs of this result. The first one relies on the existence of the Dieudonné (non-commutative) determinant (cf. [1, p. 165]). As in Lemma 8, it suffices to show $N\left(\mathrm{GL}_{n}(D)\right) \subset N\left(D^{\times}\right)$. The reduced norm map $N: D^{\times} \rightarrow K^{\times}$factors through the map nr: $D^{\#} \rightarrow K^{\times}$, where $D^{\#}:=$ $D^{\times} /\left[D^{\times}, D^{\times}\right]$. Then there is a group homomorphism (called the Dieudonné determinant)

$$
\operatorname{det}: \operatorname{GL}_{n}(D) \rightarrow D^{\#}
$$

such that $N(a)=\operatorname{nr}(\operatorname{det} a), \forall a \in \mathrm{GL}_{n}(D)$. It follows that $N\left(\mathrm{GL}_{n}(D)\right) \subset$ $N\left(D^{\times}\right)$.

Recall an elementary matrix in $\operatorname{Mat}_{n}(D)$ is a matrix $E=\left(e_{i j}\right)$ whose diagonal entries $e_{i i}$ are 1 and off diagonal entries $e_{i j}(i \neq j)$ are all 0 except for one. Let $E_{n}(D) \subset \mathrm{GL}_{n}(D)$ be the subgroup generated by all elementary matrices. Using the row and column reductions, for any $X \in \mathrm{GL}_{n}(D)$, there are elements $P, Q \in E_{n}(D)$ such that $P X Q=\operatorname{diag}\left(a_{1}, \ldots, a_{n}\right)$, where 
$a_{i} \in D^{\times}$. It is easy to show that elements $\operatorname{diag}\left(a, a^{-1}, 1, \ldots, 1\right)$ are contained in $E_{n}(D)$. Thus, the group $\mathrm{GL}_{n}(D)$ is generated by $E_{n}(D)$ and the subgroup $\left\{\operatorname{diag}(1, \ldots, 1, a) \mid a \in D^{\times}\right\}$. Since $N\left(E_{n}(D)\right)=1$, we get $N\left(\operatorname{GL}_{n}(D)\right)=$ $N\left(D^{\times}\right)$.

Remark 10. One can show a slightly stronger result of Lemma 8 that the Lie group $\mathrm{GL}_{n}(\mathbb{H})$ is connected. The kernel $G_{1}$ of the reduced norm map $N: \mathrm{GL}_{n}(\mathbb{H}) \rightarrow \mathbb{R}^{\times}$is the group of $\mathbb{R}$-points of a connected, semi-simple and simply connected algebraic $\mathbb{R}$-group. Thus, $G_{1}$ is connected. Then the fibers of the reduced norm map $N$ are all connected as they are principal homogeneous spaces under $G_{1}$. As the image of the map $N$ is also connected (Lemma 8), the Lie group $\mathrm{GL}_{n}(\mathbb{H})$ is connected.

\section{Strong Approximation}

In this section we give a short exposition of the strong approximation theorem, which serves as the key ingredient in the proof of Theorem 3 , We keep the notation of Section 3. In particular $K$ denotes a global field and $S$ is a non-empty finite set of places of $K$.

Recall that a connected semi-simple algebraic group $G$ over a field $k$ is said to be simply connected if there is no finite surjective homomorphism $f: G^{\prime} \rightarrow G$ whose kernel is non-trivial.

Theorem 11 (The strong approximation theorem). Let $G$ be a connected, semi-simple and simply connected algebraic group over $K$. Suppose that

(*) $G$ does not contain any $K$-simple factor $H$ such that the topological group $H_{S}:=\prod_{v \in S} H\left(K_{v}\right)$ is compact.

Then the group $G(K)$ is dense in $G\left(\mathbb{A}_{K}^{S}\right)$.

Proof. See Kneser [7] when $K$ is a number field and Prasad [8] when $K$ is a global function field. The results were proved based on the validity of the Hasse principle, i.e. the map

$$
H^{1}(K, G) \rightarrow \prod_{v} H^{1}\left(K_{v}, G\right)
$$


is injective. The Hasse principle was known to hold for any simply-connected group at that time except possibly for those of type $E_{8}$. The last case (type $\left.E_{8}\right)$ was finally completed by Chernousov in 1989.

The strong approximation theorem is a strong version of "class number one" result.

Corollary 12. Let $G$ be as in Theorem 11 satisfying the condition (*) and assume that $S$ contains all archimedean places of $K$. Then for any open compact subgroup $U \subset G\left(\mathbb{A}_{K}^{S}\right)$, the double coset space $G(K) \backslash G\left(\mathbb{A}_{K}^{S}\right) / U$ consists of a single element.

Let $A, C$ and $R$ be as in Section 3. Now we let $G$ and $\underline{C}^{\times}$denote the algebraic groups $K$ associated to the multiplicative groups of $A$ and $C$, respectively. For any commutative $K$-algebra $L$, one has

$$
G(L)=\left(A \otimes_{K} L\right)^{\times}, \quad \underline{C}^{\times}(L)=\left(C \otimes_{K} L\right)^{\times} .
$$

We denote again by $N: G \rightarrow \underline{C}^{\times}$the homomorphism of algebraic $K$-groups induced by the reduced norm map $N: A \rightarrow C$, and let $G_{1}:=\operatorname{ker} N$ denote the reduced norm-one subgroup. It is easy to see that the base change $G_{1} \otimes \bar{K}$ is a finite product of simple groups of the form $\mathrm{SL}_{m}$, and hence $G_{1}$ is semi-simple and simply connected.

Recall that $A$ is said to satisfy the Eichler condition with respect to $S$, if for any simple factor $A_{i}$ of $A$ there is one place $w$ of the center $K_{i}$ over some place $v$ in $S$ such that the completion $A_{i, w}$ at $w$ is not a division $K_{i, w^{-}}$ algebra. Another way to rephrase the last condition for $A_{i}$ is that the kernel of the reduced norm map

$$
N_{A_{i} / K_{i}}: \prod_{v \in S}\left(A_{i} \otimes K_{v}\right)^{\times} \rightarrow \prod_{v \in S}\left(K_{i} \otimes K_{v}\right)^{\times}
$$

is not compact. In other words, the algebra $A$ satisfies the Eichler condition with respect to $S$ (also denote $A=$ Eichler $/ R$, where $R$ is the ring of $S$ integers of $K$ ) if and only if the reduced norm-one subgroup $G_{1}$ satisfies the condition $(*)$ in Theorem 11, In particular, we have the following special case of Theorem 11 for $G_{1}$. 
Theorem 13. Let $A$ be a separable $K$-algebra and $G_{1}$ the associated reduced norm-one subgroup defined as above. If A satisfies the Eichler condition with respect to $S$, then $G_{1}(K)$ is dense in $G_{1}\left(\mathbb{A}_{K}^{S}\right)$.

Theorem 13 is what we use in the proof of Theorem 3. When $K$ is a number field, this is the first case of the strong approximation theorem, proved by Eichler [4]. Swan [13] gives a more elementary proof of this theorem.

Lemma 14. Suppose that $A=$ Eichler $/ R$, and let $U$ be an open compact subgroup of $G\left(\mathbb{A}_{K}^{S}\right)=\widehat{A}^{\times}$. Then the induced surjective map

$$
N: G(K) \backslash G\left(\mathbb{A}_{K}^{S}\right) / U \rightarrow N(G(K)) \backslash \widehat{C}^{\times} / N(U)
$$

is also injective.

Proof. Let $\hat{c} \in \widehat{C}^{\times}$be an element and $\hat{g} \in \widehat{A}^{\times}$with $N(\hat{g})=\hat{c}$. Then the fiber of the class $[\hat{c}]$ is

$$
N^{-1}([\hat{c}])=G(K) \backslash G(K) G_{1}\left(\mathbb{A}_{K}^{S}\right) \hat{g} U / U
$$

If $x_{1}, x_{2} \in G_{1}\left(A_{K}^{S}\right)$ be two elements, then

$$
G(K) x_{1} \hat{g} U=G(K) x_{2} \hat{g} U \Longleftrightarrow G_{1}(K) x_{1}\left(\hat{g} U \hat{g}^{-1}\right)=G_{1}(K) x_{2}\left(\hat{g} U \hat{g}^{-1}\right) .
$$

Thus, we get a surjective map

$G_{1}(K) \backslash G_{1}\left(\mathbb{A}_{K}^{S}\right) / G_{1}\left(\mathbb{A}_{K}^{S}\right) \cap \hat{g} U \hat{g}^{-1} \rightarrow G(K) \backslash G(K) G_{1}\left(\mathbb{A}_{K}^{S}\right) \hat{g} U / U=N^{-1}([\hat{c}])$.

As we know the source of (4.4) consists of one element (Corollary 12), the fiber $N^{-1}([c])$ also consists of one element.

\section{Proof of Theorem 3}

For any integer $n \geq 1$ and any ring $L$ not necessarily commutative, let $\operatorname{Mat}_{n}(L)$ denote the matrix ring over $L$, and let $\mathrm{GL}_{n}(L)$ denote the group of units in $\operatorname{Mat}_{n}(L)$. Let $N_{n}: \operatorname{Mat}_{n}(A) \rightarrow C$ be the reduced norm map, which induces a surjective homomorphism $N_{n}: \operatorname{GL}_{n}(\widehat{A}) \rightarrow \widehat{C}^{\times}$. For any integer 
$r \geq 1$, let $I_{r} \in \operatorname{Mat}_{r}(\mathbb{Z})$ be the identity matrix. Let $\varphi_{r}: \mathrm{GL}_{n} \rightarrow \mathrm{GL}_{n+r}$ be the morphism of algebraic groups which sends

$$
a \mapsto \varphi_{r}(a)=\left(\begin{array}{cc}
a & \\
& I_{r}
\end{array}\right) .
$$

Clearly any locally free right $\Lambda$-module $M$ of rank $n$ is isomorphic to a $\Lambda$-submodule in $A^{n}$. Therefore, the set $\operatorname{LF}_{n}(\Lambda)$ is in bijection with the set of global equivalence classes of the genus of the standard lattice $\Lambda^{n}$ in $A^{n}$. The latter is naturally isomorphic to $\mathrm{GL}_{n}(A) \backslash \mathrm{GL}_{n}(\widehat{A}) / \mathrm{GL}_{n}(\widehat{\Lambda})$. If $n \geq 2$, then by Lemma 14 the induced map

$$
N_{n}: \mathrm{GL}_{n}(A) \backslash \mathrm{GL}_{n}(\widehat{A}) / \mathrm{GL}_{n}(\widehat{\Lambda}) \stackrel{\sim}{\longrightarrow} \widehat{C}^{\times} / N_{n}\left(\mathrm{GL}_{n}(A)\right) N_{n}\left(\mathrm{GL}_{n}(\widehat{\Lambda})\right)
$$

is a bijection.

Lemma 15. We have

$$
\widehat{C}^{\times} / N_{n}\left(\operatorname{GL}_{n}(A)\right) N_{n}\left(\mathrm{GL}_{n}(\widehat{\Lambda})\right)=\widehat{C}^{\times} / N\left(A^{\times}\right) N\left(\widehat{\Lambda}^{\times}\right)=\widehat{C}^{\times} / C_{+, A}^{\times} N\left(\widehat{\Lambda}^{\times}\right) .
$$

Proof. We have seen in Lemma 7 that $N_{n}\left(\mathrm{GL}_{n}(A)\right)=N\left(A^{\times}\right)=C_{+, A}^{\times}$. We now prove $N_{n}\left(\mathrm{GL}_{n}\left(\Lambda_{v}\right)\right)=N\left(\Lambda_{v}^{\times}\right)$for $v \notin S$ since the statement is local. The group $\operatorname{GL}_{n}\left(\Lambda_{v}\right)$ contains as a subgroup the group $E_{n}\left(\Lambda_{v}\right)$ of elementary matrices with values in $\Lambda_{v}$. Since $\Lambda_{v}$ is semi-local, we have a result of Bass [12, Proposition 8.5] that $\mathrm{GL}_{n}\left(\Lambda_{v}\right)$ is generated by the subgroup $E_{n}\left(\Lambda_{v}\right)$ and the image $\varphi_{n-1}\left(\mathrm{GL}_{1}\left(\Lambda_{v}\right)\right)$. Since $E_{n}\left(\Lambda_{v}\right)$ is contained in the kernel of $N_{n}$, we have $N_{n}\left(\operatorname{GL}_{n}\left(\Lambda_{v}\right)\right)=N_{n}\left(\varphi_{n-1}\left(\Lambda_{v}^{\times}\right)\right)=N\left(\Lambda_{v}^{\times}\right)$.

For any integer $r \geq 1$, we say two locally free right $\Lambda$-ideals $J$ and $J^{\prime}$ are r-stably isomorphic if $J \oplus \Lambda^{r} \simeq J^{\prime} \oplus \Lambda^{r}$ as $\Lambda$-modules. Let $\hat{c} \in \widehat{A}^{\times}$be an element such that $\hat{c} \Lambda=J$; we have $\varphi_{r}(\hat{c}) \Lambda^{r+1}=J \oplus \Lambda^{r}$.

The morphism $\varphi_{r}$ induces the following commutative diagram:

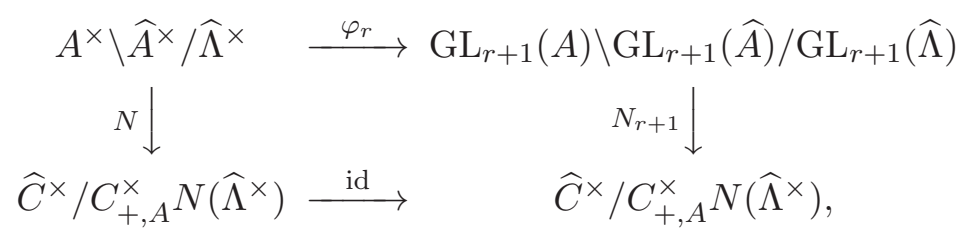


where the reduced norm map $N_{r+1}$ is known be a bijection. Two isomorphism classes $[J]$ and $\left[J^{\prime}\right]$ in $A^{\times} \backslash \widehat{A}^{\times} / \widehat{\Lambda}^{\times}$are $r$-stably isomorphic if and only if $\varphi_{r}([J])=\varphi_{r}\left(\left[J^{\prime}\right]\right)$. As $N_{r+1}$ is an isomorphism, this is equivalent to $N([J])=N\left(\left[J^{\prime}\right]\right)$. The latter condition is independent of $r$. Therefore, we conclude the following statement.

Lemma 16. Let $J$ and $J^{\prime}$ be two locally free right $\Lambda$-ideals. The following statements are equivalent.

(1) $J$ and $J^{\prime}$ are stably isomorphic.

(2) $J$ and $J^{\prime}$ are $r$-stably isomorphic for some $r \geq 1$.

(3) $J$ and $J^{\prime}$ are $r$-stably isomorphic for all $r \geq 1$.

(4) One has $N([J])=N\left(\left[J^{\prime}\right]\right)$ in $\widehat{K}^{\times} / C_{+, A}^{\times} N\left(\widehat{\Lambda}^{\times}\right)$.

Thus, the reduced norm map $N$ induces an isomorphism

$$
\nu: \operatorname{Cl}(\Lambda) \simeq \widehat{C}^{\times} / C_{+, A}^{\times} N\left(\widehat{\Lambda}^{\times}\right)
$$

We now check that $\nu$ is a group homomorphism. Let $J$ and $J^{\prime}$ be two locally free $\Lambda$-ideals. Let $\hat{c}$ and $\hat{c}^{\prime}$ be elements in $\hat{A}^{\times}$such that $\hat{c} \Lambda=J$ and $\hat{c}^{\prime} \Lambda=J^{\prime}$. Put $J^{\prime \prime}:=\hat{c} \hat{c}^{\prime} \Lambda$. We claim that

(a) $J \oplus J^{\prime} \simeq J^{\prime \prime} \oplus \Lambda$ as $\Lambda$-modules;

(b) $\nu\left([J]_{s}\right) \nu\left(\left[J^{\prime}\right]_{s}\right)=\nu\left(\left[J^{\prime \prime}\right]_{s}\right)$.

Statement (a) follows from

$$
\left[\begin{array}{cc}
\hat{c} \hat{c}^{\prime} & 0 \\
0 & 1
\end{array}\right] \cdot \Lambda^{2}=J^{\prime \prime} \oplus \Lambda, \quad \text { and } \quad N_{2}\left(\left[\begin{array}{cc}
\hat{c} \hat{c}^{\prime} & 0 \\
0 & 1
\end{array}\right]\right)=N_{2}\left(\left[\begin{array}{cc}
\hat{c} & 0 \\
0 & \hat{c}^{\prime}
\end{array}\right]\right)
$$

in $\widehat{C}^{\times} / C_{+, A}^{\times} N\left(\widehat{\Lambda}^{\times}\right)$. Statement (b) follows from

$$
\nu\left([J]_{s}\right) \nu\left(\left[J^{\prime}\right]_{s}\right)=N([\hat{c}]) N\left(\left[\hat{c}^{\prime}\right]\right)=N\left(\left[\hat{c} \hat{c}^{\prime}\right]\right)=\nu\left(\left[J^{\prime \prime}\right]_{s}\right) .
$$

This completes the proof of Theorem 3 . 


\section{Acknowledgments}

The manuscript is prepared while the author's stay at the Max-PlanckInstitut für Mathematik in Bonn. He is grateful to the Institute for kind hospitality and excellent working environment. The author thanks JK Yu and Ming-Chang Kang for pointing out the references [8] and [14] to him. The author is partially supported by the grants MoST 100-2628-M-001-006MY4 and 103-2918-I-001-009.

\section{References}

1. C. W. Curtis and I. Reiner, Methods of representation theory. Vol. I. With applications to finite groups and orders, Pure and Applied Mathematics. A Wiley-Interscience Publication. John Wiley \& Sons, Inc., New York, 1981, 819 pp.

2. Charles W. Curtis and I. Reiner, Methods of representation theory. Vol. II. With applications to finite groups and orders, Pure and Applied Mathematics (New York). A Wiley-Interscience Publication. John Wiley \& Sons, Inc., New York, 1987, 951 pp.

3. M. Eichler, Über die Idealklassenzahl total definiter Quaternionenalgebren, Math. Z., 43 (1938), 102-109.

4. M. Eichler, Allgemeine Kongruenzklasseneinteilungen der Ideale einfacher Algebren über algebraischen Zahlkörpern und ihre L-Reihen, J. Reine Angew. Math., 179 (1938) $227-251$.

5. A. Fröhlich, Locally free modules over arithmetic orders, J. Reine Angew. Math., 274/275 (1975), 112-124.

6. Emmanuel Hallouin and Christian Maire, Cancellation in totally definite quaternion algebras, J. Reine Angew. Math., 595 (2006), 189-213.

7. Martin Kneser, Strong approximation, Algebraic Groups and Discontinuous Subgroups (Proc. Sympos. Pure Math., Boulder, Colo., 1965) (1966), 187-196, Amer. Math. Soc., Providence, R.I.

8. G. Prasad, Strong approximation for semi-simple groups over function fields, Ann. Math. (2), 105 (1977), no. 3, 553-572.

9. I. Reiner, I. Maximal orders. London Mathematical Society Monographs. New Series, 28. Oxford University Press, Oxford, 2003, 395 pp.

10. Daniel Smertnig, A note on cancellation in totally definite quaternion algebras, $J$. Reine Angew. Math., 707 (2015), 209-216.

11. R. G. Swan, Projective modules over group rings and maximal orders, Ann. Math. (2), 76 (1962), 55-61.

12. R. G. Swan, K-theory of finite groups and orders, Lecture Notes in Math., vol. ,, Springer-Verlag 149 (1970), 237 pp. 
13. R. G. Swan, Strong approximation and locally free modules, Ring theory and algebra, III, pp. 153-223, Lecture Notes in Pure and Appl. Math., 55, Dekker, New York, 1980.

14. Richard G. Swan, Projective modules over binary polyhedral groups, J. Reine Angew. Math., 342 (1983), 66-172.

15. Vignéras, Marie-France, Simplification pour les ordres des corps de quaternions totalement définis, J. Reine Angew. Math., 286/287 (1976), 257-277. 